\title{
OPTICAL IMMUNE BIOSENSORS BASED ON THE NANOSTRUCTURED SILICON AND INTENDED FOR THE DIAGNOSTICS OF RETROVIRAL BOVINE LEUCOSIS
}

\author{
N. F. Starodub ${ }^{1,2}$, J. A. Sitnik ${ }^{1}$, M. M. Mel'nichenko ${ }^{2}$, O. M. Shmyryeva $^{3}$ \\ ${ }^{1}$ National University of Life and Environmental Sciences, 15 Herojev Oboroni Str., Kyiv, 03041 Ukraine; \\ nikstarodub@yahoo.com \\ ${ }^{2}$ Taras Shevchenko Kiev National University, 2 Prospect Glushkova, Kyiv, 03127, Ukraine \\ ${ }^{3}$ National Technical University, “KPI”, 37 Prospect Peremogy, Kyiv, 03056, Ukraine
}

Summery. We used the boron doped single-crystal silicon with the square wafers about $0.3 \mathrm{~mm}$ of thickness and with the resistance of $1 \mathrm{Ohm}^{*} \mathrm{~cm}$. sNPS layers were prepared by the chemical way and their thickness were changed from 3 up to $60 \mathrm{~nm}$. The parameters of the technological process at the chemical modification of the single-crystal silicon surface were controlled by the scanning electronic microscopy (SEM) and the chemical content - Auger electronic spectroscopy. Contacts were formed by the magnetron sputtering of In iones with the use of a metal mask. At the formation of the immune complex (retrovirus proteins - specific antibodies - Ab) in case of the illumination It was stated that the photosensitivity of the structures nano-porous silicon (sPNS) is a little decreased after the immobilization of antigen (Ag -crude sample of the retroviral proteins) but at the addition of Ab (serum blood of ill cows) in the dilution of 1:5000 and, particular, in 1:1000 it sharply is decreased. Unfortunately at the less level of blood dilution (from 1:100 to 1:1) the photosensitivity starts to decrease up to initial level. Maybe, it connected with the increasing of the density of the solution to be analyzed or with other mechanism of the electronic exchanges between the immune complex and the sNPS surface. The deposition of the retroviral proteins on the sNPS a few increases the photoluminescence $(\mathrm{PhL})$ level but at the formation of the specific immune complex it decreases. Moreover, the level of the $\mathrm{PhL}$ decreasing depends on the concentration of the specific $A b$ in the blood. If we used the non-specific $A b$ or the serum bovine albumin as $\mathrm{Ag}$ the level of the PhL does not change. According to our opinion the red $\mathrm{PhL}$ may be connected with the tunnel mechanism of the recombination of the charge bearers at the excitation of them in the nanocrystallites of oxide or interface. We do not exclude the hydrogen role too for the generation of the PhL extinguishing. These conclusions are as result of the coincidence of the possible reasons for the PhL decreasing in case of the immune complex formation no the sNPS surface. To them belong: a) the changes of the absorbance in the solution at the formation of the specific immune complex on the sNPS surface, b) the effect of the immune components or their interaction on the recombinant process of the photocurrent charge in the sNPS. As it is very known the light absorption in the wavelength of the excitation $(\lambda=350 \mathrm{HM})$ and in the wide field of the sNPS PhL is absent in the Ab and Ag solutions as well as in their complexes.

Key words: Silicon, nanostructured, immune biosensor, leucosis, diagnostics.

\section{Introduction.}

Bovine leucosis - is the highly foetal neoplasia of the cattle characterised by the abnormality maturation process of the blood cells and the aggregation of the neaplastic lymphocytes in the lymph nodes [1]. Clinical signs are most commonly associated with the infection and they include weight loss, decreased milk production, lymphadenopathy and posterior paresis. Virus of the type C (retrovirus family from an oncovirus rod) is the causative agent of the disease. Virus contains the revertase and six antigen proteins, among of which is the superficial (an envelope protein) glycoprotein (gp51) and the inside protein (p21) [2].

Once acquired, viral infection is lifelong and is spread by the contact between the animals. At any age animals can be infected with bovine leukaemia virus (BLV), but tumour (lymphosarcomas) is seen typically in the animals over 3 years old. Infections are usually sub clinical; only $30-70 \%$ of the infected cattle develop the persistent lymphocytes and only $0.1-10 \%$ - develop the tumours [3].

The Epidemiological and the End Results Program of USA were used to perform a descriptive epidemiological study of the leukaemia in lowa. The ecological relationships between the human leukaemia, the livestock populations and the bovine lymphosarcoma were investigated. There is a high positive correlation between the acute lymphoid leukaemia in the males and the cattle density. This relationship is higher for the dairy cattle than for the beef cattle. So, leukaemia is the potential problem from the both public health and economic perspectives [4]. 
Diagnosis of the BLV infection based on the clinical signs alone is difficult because of the wide range of symptoms. More then 20 various variants from the haematological, histological [1] and up to use of the polymerise chain reaction (PCR) $[5,6]$ as well as a different variants of the immunological methods, for example, the classical fluorescence [7], the immune diffusion [8], the radioimmune analysis [9], the immunoblot [5] were developed. The traditional immune methods have high specificity and sensitivity, but they take a lot of time, and require additional parameters such as the labelled molecules. This drawback can be overcome with the use of the modern instrumental analytical devices based on the biosensor technology.

Early we have developed [10-12] biosensor based on the surface plasmon resonance (SPR) for the express revealing of some biochemical quantities. A special attention was paid the application of this approach for the diagnostics of BLV [13-16]. This sensor showed high working characteristics in the respect of the sensitivity, simplicity and rapidity of the analysis fulfilment [17]. Nevertheless, it has some disadvantages and at first they connected with the high cost of the chips and the necessity to use not simple procedure of the preliminary transducer surface treatment from one site. From other site the SPR recorder has very high price and don't allow fulfilling a number repeated analysis. To overcome some of these disadvantages we try to apply others types of the optical biosensors for solving of the problem of the express biochemical diagnostics of BLV. Among others such biosensors that based on the sNPS attract an especial attention. Similar biosensors based on the sNPS were developed early by us for the control of the myoglobin level in the blood and for the monitoring of the bacterial protein in the air of environment [18-22]. The registration of the specific signal was made by the measurement of the changes of the PhL intensity. This approach met the practice requirements regarding the simplicity, sensitivity, selectivity and rapidity of the analysis fulfilment but the stability of the sNPS was very low and the signal registration demanded complicated device. To continue our investigations in this field we proposed a new variant of biosensor based on the sNPS

\section{Experimental.}

General abilities of sNPS. The main problem which should be solved at the application of the sNPS as the transducer in the biosensors is providing of the PhL stability during long time. It depends on the sNPS structure, the content of the inter phase layers and the porosity level. These abilities of the sNPS may be determined by the method and the regimes of its forming. Our investigations of the sNPS shown that the samples prepared by the method of the chemical etching have the stable ChL, conductivity and photoconductivity characteristics which were preserved during 5 years.

Obtaining of sNPS by the chemical etching. This method is simpler then the galvanic anodizing and it allows forming more thin (less then $1 \mu \mathrm{m}$ ) homogenous layers of the sNPS. This method is based on the next. At the introducing of some oxidants in the electrolyte based on the fluoric acid the self solving process for the silicon plate immersed in this solution is started without any external voltage. The electrolyte with $\mathrm{HNO}_{3}(70 \%)$ or $\mathrm{NaNO}_{2}$ as oxidants are most often used for such process at the different concentrations of fluoric acid: $\mathrm{HF}: \mathrm{HNO}_{3}: \mathrm{H}_{2} \mathrm{O}=1: 5: 10$, or $1: 3: 5$, or $4: 1: 5$, or $\mathrm{HF}: \mathrm{NaNO}_{2}=100 \mathrm{ml}: 2 \mathrm{~g}$. Cathode reaction is realized through the reduction of the oxidant by the electrons from the anode:

$$
\mathrm{HNO}_{3}+3 \mathrm{H}^{+} \rightarrow \mathrm{NO}+2 \mathrm{H}_{2} \mathrm{O}+3 \mathrm{~h}^{+} \text {. }
$$

The complete chemical reaction on the Si surface may be presented by the next equation:

$$
3 \mathrm{Si}+4 \mathrm{HNO}_{3}+18 \mathrm{HF} \rightarrow 3 \mathrm{H}_{2} \mathrm{SiF}_{6}+4 \mathrm{NO}+8 \mathrm{H}_{2} \mathrm{O}+3(4-n) \mathrm{h}^{+}+3(4-n) \mathrm{e}^{-} \text {. (2) }
$$

In opposite to the electrolytic process the chemical etching is self regulated and strong depends on the initial solution content. Oxidant at the etching plays the same role as the anode current density in the electrochemical method. It means that the higher $\mathrm{HNO}_{3}$ concentration responds to the electrochemical regime with the big current density. At the some time a big quantity of $\mathrm{HF}$ in the solution leads to process which restricted by the hole accessible and responded to the regime with the low current density in case of the electrochemical method. That is why, the content of $\mathrm{HNO}_{3}$ is the most important parameter at the chemical etching.

Position of the PhL spectra maximum does not depends on the oxidant type and is at $\lambda \sim 625 \mathrm{~nm}$. Method of the chemical etching is most adapted to the mass manufacturing and is interested for the preparation of the thin layers of the sNPS for the devices. Nevertheless, at the application of this method there is necessary to solve problems connected with the reproducibility and homogeneity of the layers.

So, due to changes of the surface state and the etching content there is possible to have the thin homogeneous luminescent layers of the porous silicon (PS) with the high level of the reproducibility obtained by the chemical method and without the application of the electrical field.

The structure of the sNPS was investigated by the scanning electronic microscopy (Fig. 1) and the chemical content - Auger spectroscopy (Fig. 2).

Spectra of the optical reflectance depend on the structure and dimensions of the nanocrystallites, air pores, level of porosity and the thickness of the layer (Fig. 3). 


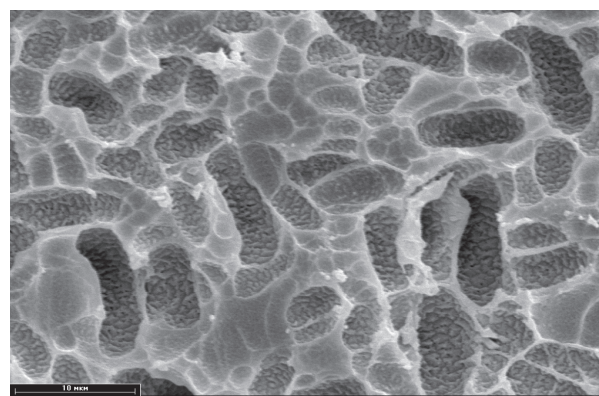

Fig. 1. General view of the whole surface of sNPS obtained by SEM.
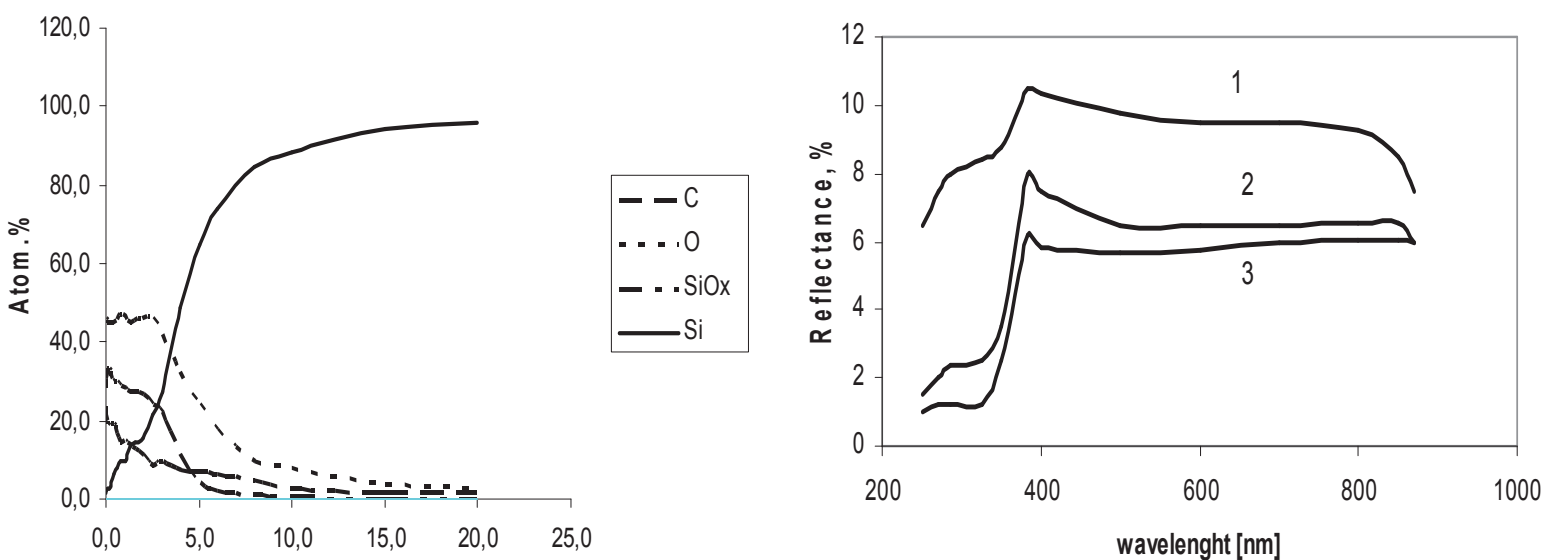

Fig. 2 (left). Dispersion of the elements in the sNPS layer (the speed of the etching $-3 \mathrm{~nm} / \mathrm{min}$ ).

Fig. 3 (right). Spectra of the optical reflectance of the sNPS layer in the dependence on the dimension of the nanocrystallites: $1-5 \mathrm{~nm}, 2-15 \mathrm{~nm}, 3-30 \mathrm{~nm}$.

The maximal photosensitivity in the visible diapason of the spectra $(30-35 \mathrm{~mA} / \mathrm{Lm})$ is typical for the sNPS layers with the dimensions of the nanocrystallites in $15 \mathrm{~nm}$ and it decreased with the growing of the last. At the same time the maximal sensitivity to the ultraviolet irradiation was obtained for the sNPS layers with the dimensions of the nanocrystallites in 20-25 nm. sNPS layers obtained by the method of the electrochemical etching as well as by the chemical etching shown the PhL abilities which were typical for this material: the wide region in the visible diapason with the intensity which is enough for the observation of the PhL with the naked eye. All samples of sNPS obtained by the chemical method had the bright irradiation with the maximum at $\lambda \sim 640 \mathrm{~nm}$ and that prepared by the electrochemical way $700 \mathrm{~nm}$. According to the measurements of the $\mathrm{PhL}$ and the photoconductivity of the prepared films it was chosen the optimal content for the chemical treatment and time for the etching.

Photoconductivity of the SNPS. Photoelectric processes in the layers of the sNPS which belong to the semiconductors materials accomplished in the result of the photo generation of the electron-holes pairs and following their dividing and recombination. The processes of adsorption on the sNPS surface may arouse a new photoelectric effects. Nanocrystallites of the silicon with the dimensions from one to dozen $\mathrm{nm}$ are as the silicon regions which are not dissolved and surrounded by the production of the electrochemical and the chemical reactions. At the dimensions less then $15-20 \mathrm{~nm}$ it is aroused the quant-dimensioned effects which lead to the quantization of the energetic spectra of the charge curriers, widening of the prohibited zone up to $1.7-3.4 \mathrm{eV}$ and to decreasing of the dielectric permeability. The luxampere characteristics of the obtained samples have two plots: the linear and the sub linear which achieves the saturation at the illumination more then 10000 lux. The samples with the nanolayer thickness of 15-18 nm have the maximal photosensitivity that has good correlation with the results of the experiments with the PhL (Fig. 4).

There is necessary to mention that the changing of the etching content and the solution concentrations brings to changing of the dynamics of NPS layer grow, the porosity level, the correlation of the dimensions of the crystallites and the holes, the chemical content and the profile of the dispersion of main admixtures. 


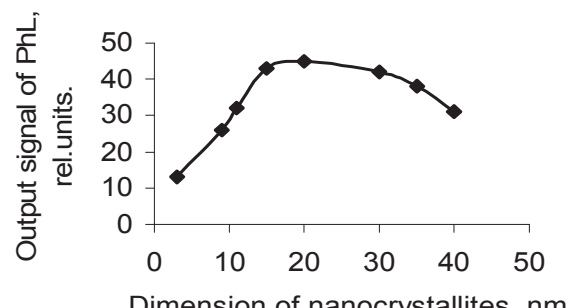

Fig. 4. Dependence of the intensity of the PhL of the sNPS layers at the $650 \mathrm{~nm}$ on the dimensions of the nanocrystallites.

Some materials and chemicals for the immune chemical analysis. The layers of the sNPS for the photoresistors were obtained by the chemical etching of the monocrystalline silicon in the solution of HF and $\mathrm{HNO}_{3}$ as described above. Preliminary it was developed the optimal way for the formation of the ohmic contact on the surface of the sNPS from alumina and indium by the magnetron sputtering with the application of the special masks. I was found that the contacts from indium are non stable due to the mechanical softness of this metal. At the measurements the contact integrity was destroyed since under it the porous surface was located. In our investigations the contacts were formed from the alumina with the thickness about $3 \mu \mathrm{m}$.

As sources of the antigens $(\mathrm{Ag})$ it was used the mixture of the retroviral proteins obtained from the joint venture of the "Leuconad" (Poltava, Ukraine). Blood serums from ill cows were kindly presented by this venture too.

Ag was dissolved in $0.05 \mathrm{M}$ tris- $\mathrm{HCl}$ buffer $(\mathrm{pH} 7,3)$ at the different concentration.

Registration of the specific immune complex by the determination of sPNS photoconductivity. At the beginning the specific $\mathrm{Ab}$ in the volume of $1 \mu \mathrm{l}$ was placed on the photoresistor surface between the contacts (Fig. 5). Then this solution was evaporated at the room temperature or at the air stream.

The direct voltage $(5 \mathrm{~V})$ from the stabilized power supply was applied to the ohmic contacts and the current was measured by the digital voltmeter of B7-35 type at the absence of lighting (dark regime) as well as the photocurrent (the difference between the light and dark currents) was registered at the lightening of the sensitive surface by the white spectrum light (source A, illumination of 7000 lux). At the drawing of Ag layer on the sensitive plate and after its drying the measurements of the dark and light current were repeated. These measurements were made after the immune complex formation (interaction of $\mathrm{Ag}$ with specific $\mathrm{Ab}$ in the serum blood) too. The control of the reaching of the sensor initial state was done according to the reduction of the dark current value after washing of the sensitive surface by the buffer solution. The time of the single analysis was 5-10 min only.

Registration of the specific immune complex by the PhL of the sPNS. Design of the prototype includes the source of the ultraviolet (UV) radiation (1) with the wavelength of $350 \mathrm{~nm}$, two photodiodes (2 and 3) based on the mono crystalline silicon and placed at the angle of $20-25^{\circ}$ relatively to the plate with the layer of the sNPS (4) and the photo diode (5) intended for the determination of the incident UV (Fig. 6). At the adsorption of the biological molecules the level of the PhL of the sPNS and the output of the voltage of the consecutive connected photo registers are decreasing. Application of two photo registers of the $\mathrm{PhL}$ increases the sensitivity.
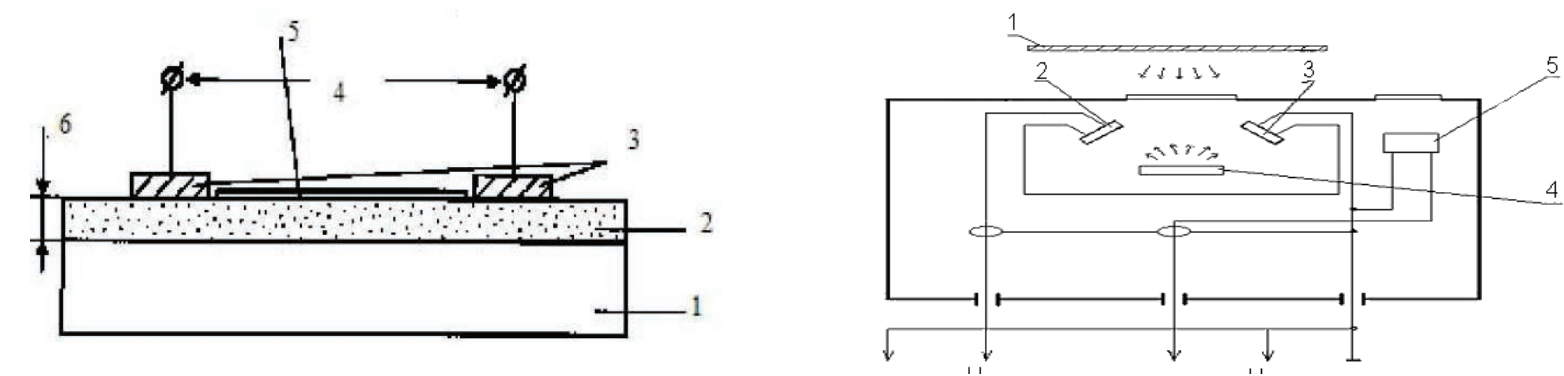

. Fig. 5 (left). Scheme of the photoresistor structure based on the sNPS and intended for the analysis of the interactions between biological structures. Where: 1 - the crystalline silicon, 2 - the sNPS, 3 - the electrical contacts (Al with the thickness of $\sim 3 \mu \mathrm{m}$ ), 4 - the applied voltage, 5 - the biological object, 6 - the thickness of the sNPS of $10-40 \mathrm{~nm}$.

Fig. 6 (right). Design of PhL biosensor (see explanation in the text). 
To take into attention the possible changing of the incident UV the photodiode (5) is used. The output signal is determined as the relation between the output signal from the photodiodes of 2 and 3 and the output signal from the photodiode of 5 . Photodiodes of the $n-p-p^{+}$-structures work in the photo generative regime. Such construction is related to the systems of the differential type

\section{Results and discussin.}

Determination of the retroviral specific Ab in the blood of the ill cow by the measurement of the sPNS photoconductivity. It was stated that the photosensitivity of the sPNS is a little decreased after the immobilization of $\mathrm{Ag}$ (crude sample of the retroviral proteins) but at the addition of Ab (serum blood of ill cows) in the dilution of 1:5000 and, particular, in 1:1000 it sharply is decreased. Unfortunately at the less level of blood dilution (from 1:100 to 1:1) the photosensitivity starts to decrease up to initial level. Maybe, it connected with the increasing of the density of the solution to be analyzed or with other mechanism of the electronic exchanges between the immune complex and the sNPS surface.

If we taken the blood serum from not ill cow the level of the photo current did not change in the comparison with the initial one. The same situation was observed if we used the bovine serum albumin instead of the crude samples of the retroviral proteins.

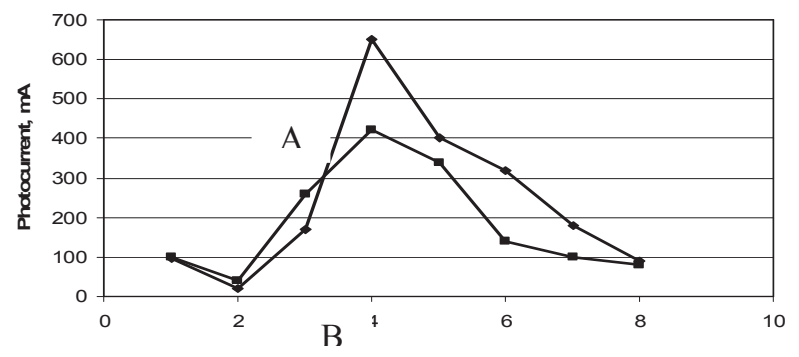

Fig. 7. The level of the photocurrent before (1) and after deposition of $\mathrm{Ag}$ (2) and $\mathrm{Ab}$ in the different concentration (3$8-1: 5000,1: 1000,1: 100,1: 50,1: 10$ and 1:1, respectively). Line $A$ and $B$ are respect to two different samples of blood serum.

So, the experimental results give us possibility to consider that the application of the proposed principle of the immune biosensor may be very perspective for the different types of the biochemical diagnostics not for revealing of the retroviral infection in cow only. Of course there is necessary to understand what kind are influences of the high protein concentration on the process of the recombination in the SPNS. There is necessary to underline that the overall time of the analysis is several dozen minutes only instead of several hours in case of the traditional used ELISA-method or several days at the realization of the immune diffusion test.

Determination of the retroviral specific Ab in the blood of the ill cow by the method of the PhL of the sPNS. The deposition of the retroviral proteins on the sPNS a few increases the PhL level but at the formation of the specific immune complex it decreases. Moreover, the level of the $\mathrm{PhL}$ decreasing depends on the concentration of the specific $\mathrm{Ab}$ in the blood (Fig. 8). If we used the non-specific $\mathrm{Ab}$ or the serum bovine albumin as $\mathrm{Ag}$ the level of the $\mathrm{PhL}$ does not change.

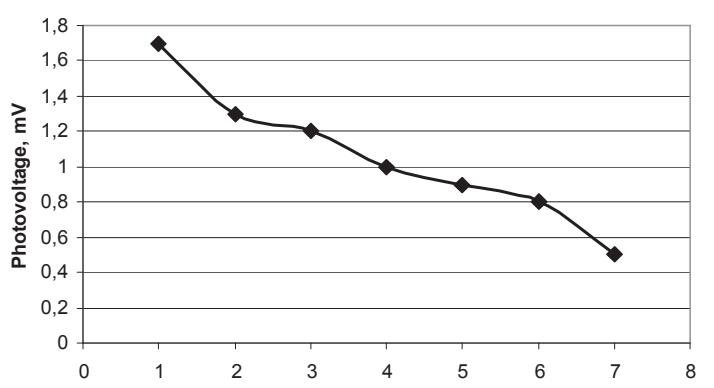

Fig. 8. Dependence of the PhL intensity on the concentration of the specific $A b$ in the serum blood of ill animals. Abscissa - 1 - immobilized Ag; 2-7 - dilution of blood serum: 1:5000; 1:1000; 1:100; 1:50; 1:10; 1:1, respectively.

According to our opinion the red $\mathrm{PhL}$ may be connected with the tunnel mechanism of the recombination of the charge bearers at the excitation of them in the nanocrystallites of oxide or interface. We do not exclude the hydrogen role too for the generation of the $\mathrm{PhL}$ extinguishing. These conclusions are as result of the coincidence of the possible reasons for the PhL decreasing in case of the immune complex formation no the sNPS surface. To them belong: a) the changes of the absorbance in the solution at the formation of the specific immune complex on the sNPS surface, b) the effect of the immune components or their interaction on the recombinant process of the photocurrent charge in the 
sNPS. As it is very known the light absorption in the wavelength of the excitation ( $\lambda=350 \mathrm{HM})$ and in the wide field of the sNPS PhL is absent in the Ab and Ag solutions as well as in their complexes.

\section{Conclusion}

The above presented experimental data testify that the sNPS may be used as very simple transducers with the long stable their abilities at the creation of the immune biosensors. The formed specific immune complex on the sNPS surface may be registered by the measurement of its $\mathrm{PhL}$ or photoconductivity. According to the obtained results in the respect of the application of such immune biosensors for the biochemical diagnostics of the bovine leucosis it is possible to conclude that they will respond all practice demands, especially, sensitivity, simplicity, rapidity of the analysis and its fulfillment in field conditions. These biosensors may be applied for registration of any biochemical quantities which may form immune complex. Further investigations should be directed on the studying of the mechanisms of the biochemical signal registration by the sNPS and on the specification of all concrete moments of the analysis fulfillment.

\section{References}

1. Balida V, Ferrer J.F. Expression of the bovine leukemia virus and its internal antigen in blood lymphocytes. Proc. Soc. Exp. Biol. Med., 1977, 156, pp.388-391.

2. Matthews R.E.F. Classification and nomenclature of viruses. Inter Virology, 1979, 12, pp.128-296.

3. Angelino J.L., Garcia M, Birgel E.H. Productive and reproductive performance in cattle infected with bovine leukosis virus. Dairy Res., 1998, 65, pp. 693-695.

4. Donham K.J., Berg J.W., Sawin R.S. Epidemiologic relationships of the bovine population and human leukemia in lowa. Am. J. Epidemiology, 1980, 112, pp. 80-92.

5. Fechner H., Kurg A., Geue L., Blankenstein P., Mewes G., Ebner D., Beier D. Evaluation of Polymerase Chain Reaction (PCR) Application in Diagnosis of Bovine Leukemia Virus (BLV) infection in Naturally Infected Cattle. J. Vet. Med., 1996, B 43, pp. 621-630.

6. Blankenstein P., Fechner H. Possibilities and limitations for use of the polymerase chain reaction (PCR) in the diagnosis of bovine leukemia virus (BLV) infection in cattle. Dtsch. Tierarztl. Wochenschr.,1998,105,pp. 408-412.

7. Ferrer J.F., Avila L., Stock N.D. Serological detection of type C viruses found in bovine cultures. Cancer Res., 1972, 32, pp. 1864-1870.

8. Miller J., Olson C. Precipitation abtibody to an internal antigen of the C-type virus associated with bovine lymphosaroma. J. Nat .Cancer Inst., 1972, 49, p.1459-1469.

9. Franz J., Hampl J., Hofirek B., Skrobak F., Svoboda I., Granatova M. Radioimmunologic detection of antibodies to bovine leukemia virus Vet. Med. (Praha), 1986, 31, pp. 459-468.

10. Starodub N.F., Dibrova T.I., Shirshov Yu..M., Kostiukevich K.V. Development of sensor based on the surface plasmon resonance for control of biospecific interaction. In: Eurosensors 12 and $11^{\text {th }}$ Europeans Conf..on solidstate transducers. Warsaw, Poland, 21-24 September, 1997, 3, pp.1429-1432.

11. Starodub N.F., Dibrova T.L., Shirshov Yu.M., Kostjukevich K.V. Development of myoglobin sensor based on the surface plasmon resonance. Ukr. Biochem. J., 1999, 71, pp. 33-37.

12. Starodub V.M., Starodub N.F. Electrochemical and optical biosensors: origin of development, achievements and perspectives of practical application. In: NATO series "Novel processes and control technologies in the food industry". Bozoglu F et al. (ed) Amsterdam, 2001, pp. 63-94.

13. Starodub N.F., Arthjuch V.P., Pirogova L.V., Nagajeva L.I., Dobrosol G.I., Pavlenko M., Grotevich V. Express diagnostics of bovine leucosis on the basis of biosensor analysis. Vet. Med., 2001, N11, pp. 26-27.

14. Pirogova L.V, Arthjuch V.P., Starodub N.F, Nagajeva L.I., Dobrosol G.I. Express diagnostics of bovine leucosis with the help of immune sensor based on the surface plasmon resonance. Ukr. Biochem. J., 2002, 74, pp.88-92.

15. Starodub N.F., Pirogova L.V., Artyukh V.P., Starodub V.N. Biospecific interactions on the optical transducer surface the base of infection diagnostics. In: NATO ARW "Frontiers of Multifunctional Nanosystems. Buzaneva E., Scharff P. (ed), 2002, 57, pp. 369-376.

16. Starodub N.F., Starodub V.N. Infectious bovine leucosis and its diagnostics. Biopolymers and Cell., 2003, 19, pp. 307-316.

17. Starodub M.F., Romanov V.O., Kochan R.V. et al. Implementation of SPR-biosensors for express-diagnostics of acute viral infection and mycotoxicosis. Bul. Khmelnitski Nat. Univ., 2006, N6, pp. 223-226.

18. Starodub N.F., Fedorenco L.L., Starodub V.M. et al. Extinguishing of visible photoluminescence of porous silicon - stimulated by antigen-antibody immunocomplecx formation. In: Optical organic and semiconductor inorganic materials. 26-29 August 1996, Riga, Latvia, 1996, pp. 73-76.

19. Starodub V.M., Fedorenko L.L, Starodub N.F. Control of a myoglobin level in solution by the bioaffinic sensor based on the photoluminescence of porous silicon. In: Eurosensors XII and 9 th UK Conf. on sensors and their applications, 13-16 September 1998, Southampton, UK, 1998, 2, pp. 817-820.

20. Starodub V.M., Starodub N.F. Optical immune sensors for the monitoring protein substances in the air. In: Eurosensor XII and $13^{\text {th }}$ European Conf. on solid-state transducers. September 12-15 1999, The Hague, The Netherlands, 1999, pp. 181-184.

21. Starodub V.M., Fedorenko L.L., Sisetskiy A.P., Starodub N.F. Control of mioglobin level in an immune sensor based on the photoluminescence of porous silicon. Sensor and Actuators, 1999, B 58, pp. 409-414.

22. Starodub V.M., Fedorenko L.L., Starodub N.F. Optical immune sensor for the monitoring protein substances in the air. Sensor and Actuators, 2000, B 68, pp. 40-47. 